\title{
Crack Detection Method on Flexure Hinges of 3-RRR Compliant Mechanism
}

\author{
Junjie $\mathrm{Gu}^{\mathrm{a}}$, Changli Liu ${ }^{\mathrm{b},{ }^{*}}$, Xuejun Wang ${ }^{\mathrm{c}}$ and Shouzhu Hu ${ }^{\mathrm{d}}$ \\ School of Mechanical and Power Engineering, East China University of Science and Technology, \\ Shanghai 200237, China

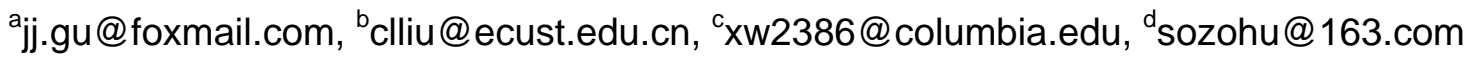

Keywords: 3-RRR Compliant Mechanism; Flexure hinge; PRBM; Crack detection.

\begin{abstract}
The planar 3-RRR compliant mechanism is a piezoelectric (PZT) actuator driven 3DOF parallel micro-motion device. It is widely used in micro-electromechanical systems, biotechnology engineering and aerospace. However, crack easily occurs on flexure hinge because alternate loading over a long period of time, which will compromise mechanism's accuracy. This paper presents a method for crack detection based on the pseudo rigid body model (PRBM). Particularly, the location of cracked hinge can be identified from the obtained force's variation on piezoelectric actuator of healthy and cracked configurations, while theirs elongation remaining the same. This research has good uses including fault diagnosis, facility for the real-time control and optimization of design of a compliant mechanism.
\end{abstract}

\section{Introduction}

The planar 3-RRR compliant mechanism is a micro motion device, which has been widely applied in micro-electromechanical systems, biotechnology engineering and aerospace area, such as assembly of micro electromechanical system and injection of the substance into minimum cells. As we know, flexure hinge is subject to an alternate loading which can easily generate crack and then eventually make the whole system fail to function or dysfunction. Thus detection of crack in the flexure hinge is important to keep the system functioning. At present, to the best of our knowledge, there is no report in literature on crack detection of the 3-RRR compliant mechanism. So it's necessary for us to do it.

Nowadays, there is an advanced crack detection method based on model. In model-based method, the fault-induced change of the system is taken into account by equivalent loads in the mathematical model. These equivalent loads are virtual forces and moments acting on the undamaged system to generate a dynamic behavior identical to that measured in the damaged system [1]. This method was first presented by Isermann [2] for fault detection of technical processes. Lees [3] presented an overview of the advances in model-based identification, from the mathematical modeling to the methods related to the different faults. His research showed that model-based method can identify crack's location, type and depth. Since there are many flexure hinges in the 3-RRR compliant mechanism, it is suitable to use model-based method to detect cracks.

Many researchers have already studied the models of 3-RRR compliant mechanism. PRBM was proposed to design and analyze a compliant mechanism based on flexure hinges [4-5]. Zou first utilized PRBM to analyze kinematic and dynamic behavior of the 3-RRR compliant mechanism [6] . Then Yong presented a method of deriving a simple, linear and effective kinematic model based on the PRBM and loop closure theory. The model is proved accurately and the methodology proposed is effective [7].

Thus this paper will present a method for crack location detection based on the PRBM. Particularly, the location of cracked hinge can be identified from the obtained force's variation on PZTs, while their elongation remaining the same. This research aims to compensate the lost accuracy introduced by cracked flexure hinge so that the mechanism may remain its original accuracy. 


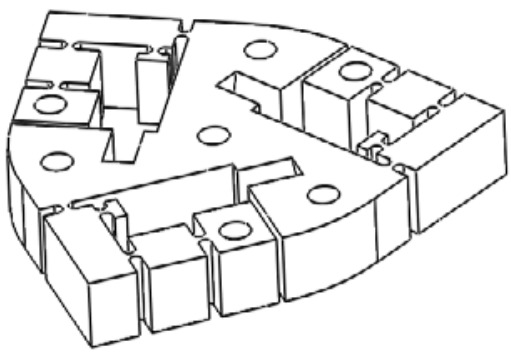

Fig.1. planar 3-RRR compliant mechanism

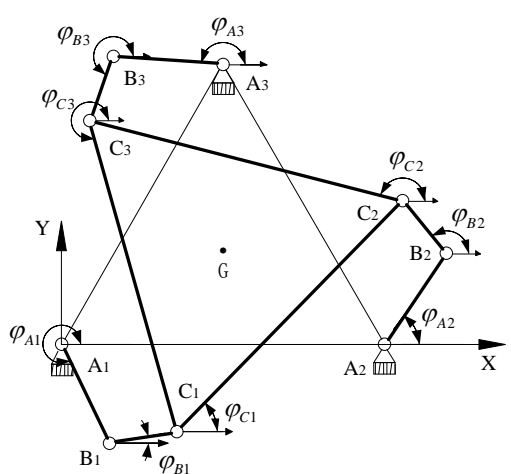

Fig.2. pseudo rigid body model

\section{Kinematics and Dynamics}

The origin of coordinate system shown in Fig.2 is point $A_{1}$ and the direction of X-axis is the same with the vector $\mathbf{A}_{\mathbf{1}} \mathbf{A}_{2}$. All the flexure hinges labeled $A_{i}$ are active joints and flexure hinges $B_{i}$ and $C_{i}$ are passive joints, therefore $\varphi_{B i}$ and $\varphi_{C i}(i=1,2,3)$ are unknowns, where $\varphi_{A i}, \varphi_{B i}$ and $\varphi_{C i}$ are the initial angular displacements measured from the direction of $\mathrm{X}$-axis. Using the loop-closure theory, those unknowns can be expressed by the input angular displacements as shown in equation (1).

$$
\left[\begin{array}{c}
\Delta \varphi_{B 1} \\
\Delta \varphi_{B 2} \\
\Delta \varphi_{B 3}
\end{array}\right]=\mathbf{H}\left[\begin{array}{c}
\Delta \varphi_{A 1} \\
\Delta \varphi_{A 2} \\
\Delta \varphi_{A 3}
\end{array}\right],\left[\begin{array}{l}
\Delta \varphi_{C 12} \\
\Delta \varphi_{C 23} \\
\Delta \varphi_{C 31}
\end{array}\right]=\mathbf{Q}\left[\begin{array}{c}
\Delta \varphi_{A 1} \\
\Delta \varphi_{A 2} \\
\Delta \varphi_{A 3}
\end{array}\right]
$$

Where $\mathrm{H}$ and $\mathrm{Q}$ are transfer matrixes of flexure hinge $B_{i}$ and $C_{i}$, respectively.

The dynamic equation of planar 3-RRR parallel compliant mechanism is given in equation (2).

$$
\mathbf{T}_{\varphi}=\mathbf{M} \ddot{\varphi}+\mathbf{K} \boldsymbol{\varphi}
$$

Where $\mathbf{T}_{\boldsymbol{\varphi}}$ represents driving torques on active joints $A_{i}(i=1,2,3) ; \boldsymbol{\varphi}$ represents the increment of angular displacements on active joints $A_{i}(i=1,2,3) ; \mathbf{M}$ is mass matrix; $\mathbf{K}$ is stiffness matrix.

Supposing there is a crack in the 3-RRR compliant mechanism and the stiffness of the torsion is reduced by $\Delta k_{b}$. Therefore the dynamic equation with crack can be expressed in equation (3) caused by the decrement of the potential energy.

$$
\mathbf{T}_{\varphi \mathrm{c}}=\mathbf{M} \ddot{\boldsymbol{\varphi}}_{\mathrm{c}}+\mathbf{K}_{\mathrm{c}} \boldsymbol{\varphi}_{\mathrm{c}}
$$

\section{Crack Detection Method}

The residual analysis of PRBM will be used between healthy configurations and cracked one to detect the location of crack. The variation of driving torque $\Delta \mathbf{T}$ can be obtained from subtracting equation (3) from equation (2)as follows.

$$
\Delta \mathbf{T}=\mathbf{T}_{\varphi}-\mathbf{T}_{\varphi c}
$$

According to the value of $\Delta \mathbf{T}$, there are three kinds of situations about the location of cracked hinges shown in equation (5). The location of a cracked flexure hinge can be determined by vector B. The algorithm of detecting the location of a cracked hinge is shown in Fig. 2.

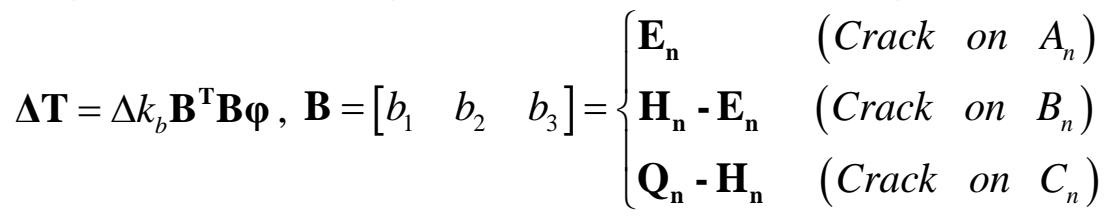

Where $\mathbf{E}_{\mathbf{n}}, \mathbf{H}_{\mathbf{n}}$ and $\mathbf{Q}_{\mathbf{n}}(n=1,2,3)$ is block matrix of $\mathbf{E}, \mathbf{H}$ and $\mathbf{Q}$ divided by row. $\mathbf{E}$ is unit matrix; $\mathbf{H}$ and $\mathbf{Q}$ are transfer matrixes of flexure hinge $B_{i}$ and $C_{i}$ shown in equation (1). 


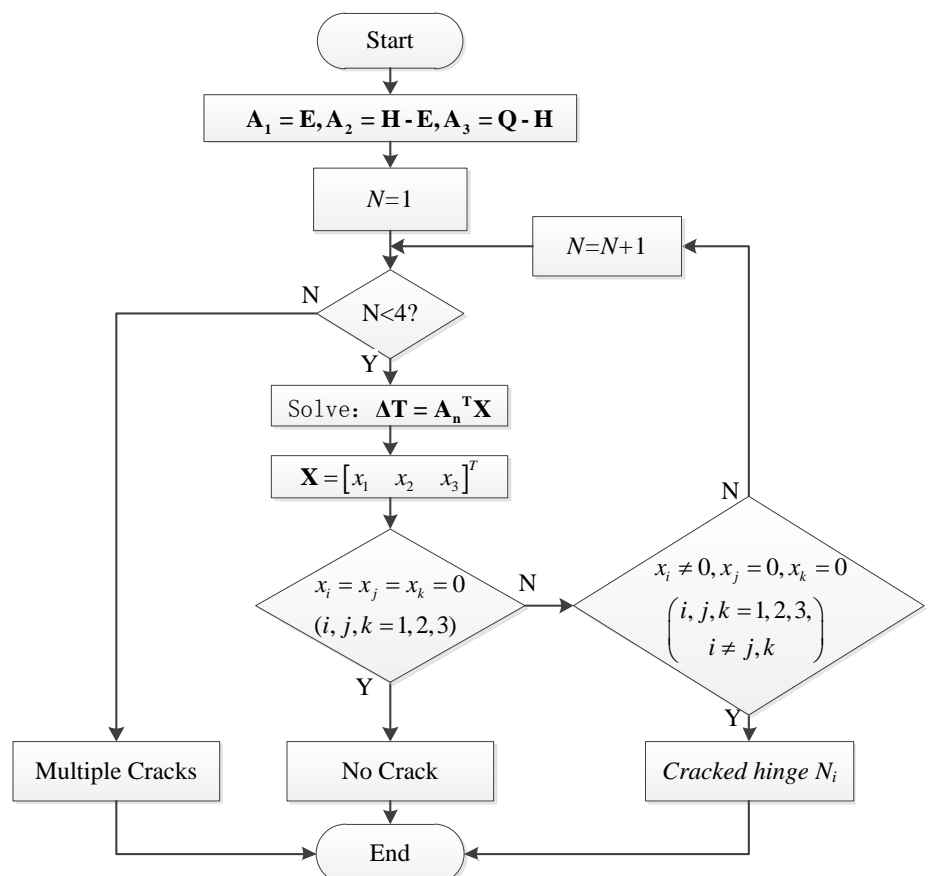

Fig.3. The algorithm of detecting the location of a cracked hinge

\section{Example for Crack Detection Method}

This section will give an example for crack detection method to approve its feasibility according to the algorithm in Fig.3 Above. The basic parameters of the prototype of planar 3-RRR compliant mechanism is shown in Table $1(i=1,2,3)$

Table 1 Basic Parameters of the planar 3-RRR compliant mechanism

\begin{tabular}{ccc}
\hline Parameters (units) & Values & Comments $(\mathrm{i}=1,2,3)$ \\
\hline $\mathrm{L} 1(\mathrm{~mm})$ & 17.22 & length of $\mathrm{AiBi}$ \\
$\mathrm{L} 2(\mathrm{~mm})$ & 11 & length of $\mathrm{BiCi}$ \\
$\mathrm{R}(\mathrm{mm})$ & 29.55 & radius of circumscribed circle of $\triangle \mathrm{C} 1 \mathrm{C} 2 \mathrm{C} 3$ \\
$\gamma 1\left(^{\circ}\right)$ & 67.23 & angle between $\mathrm{AiCi}$ and $\mathrm{BiCi}$ \\
$\gamma 2\left({ }^{\circ}\right)$ & 46.74 & angle between $\mathrm{AiCi}$ and $\mathrm{GCi}$ \\
$\mathrm{mBC}(\mathrm{kg})$ & $2.375 \times 10^{-3}$ & mass of BiCi \\
$\mathrm{m}(\mathrm{kg})$ & 0.17 & mass of $\triangle \mathrm{C} 1 \mathrm{C} 2 \mathrm{C3}$ \\
$\mathrm{IAB}\left(\mathrm{kg} \cdot \mathrm{mm}^{2}\right)$ & 0.576 & moment of inertia of AiBi \\
$\mathrm{IBC}\left(\mathrm{kg} \cdot \mathrm{mm}^{2}\right)$ & 0.0958 & moment of inertia of BiCi \\
\hline
\end{tabular}

Supposing that a crack occurs at flexure hinge $B_{1}$ in this prototype, we will detect it according to the algorithm as shown in Fig. 3. And the amplitude and frequency of the sine wave inputs are all $0.01 \mathrm{~mm}$ and $2 \mathrm{~Hz}$ respectively. The variation of driving torque between healthy and cracked configurations is shown in equation (6)

$$
\Delta \mathbf{T}=\left[\begin{array}{l}
\Delta T_{1} \\
\Delta T_{2} \\
\Delta T_{3}
\end{array}\right]=\left[\begin{array}{c}
177.75 \\
-245.11 \sin (2 t) \\
414.40 \sin (2 t)
\end{array}\right](N \cdot \mathrm{mm})
$$

Substitution of equation (6) into equation $\Delta \mathbf{T}=\mathbf{A}^{\mathrm{T}} \mathbf{X}$ and its solutions can be expressed by Fig. 4 Fig. 6 . The flexure hing $A_{i}, B_{i}$ and $C_{i}(i=1,2,3)$ are detected respectively. Among these solutions, a special condition appears in the second one that the first elements of it doesn't equal to zero and the rest almost does. It draws a conclusion that the cracked flexure hinge is B1, which matched the supposing above. Simultaneously, the amplitude of the first element is always greater than other elements in every solution, which means the variation of $\boldsymbol{\Delta} \mathbf{T}$ in the first chain is greater than in the other chains. This is closer to the reality because the first chain has one cracked hinge and it influence any other flexure hinges on it. 

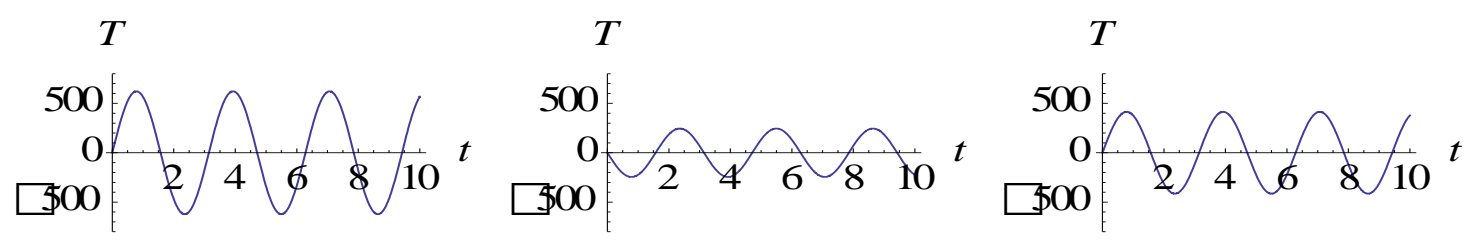

(a) $\mathrm{x} 1$

(b) $x 2$

(c) $\mathrm{x} 3$

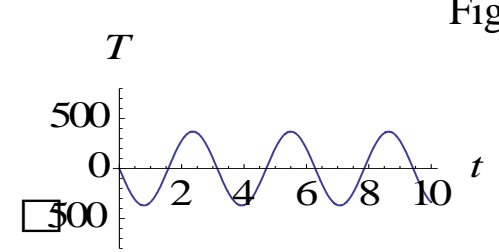

(a) $\mathrm{x} 1$

Fig.4. Solution of equation $\Delta \mathbf{T}=\mathbf{E}^{\mathrm{T}} \mathbf{X}$

$T$

Fig.5 . Solution of equation $\Delta \mathbf{T}=(\mathbf{H}-\mathbf{E})^{\mathrm{T}} \mathbf{X}$
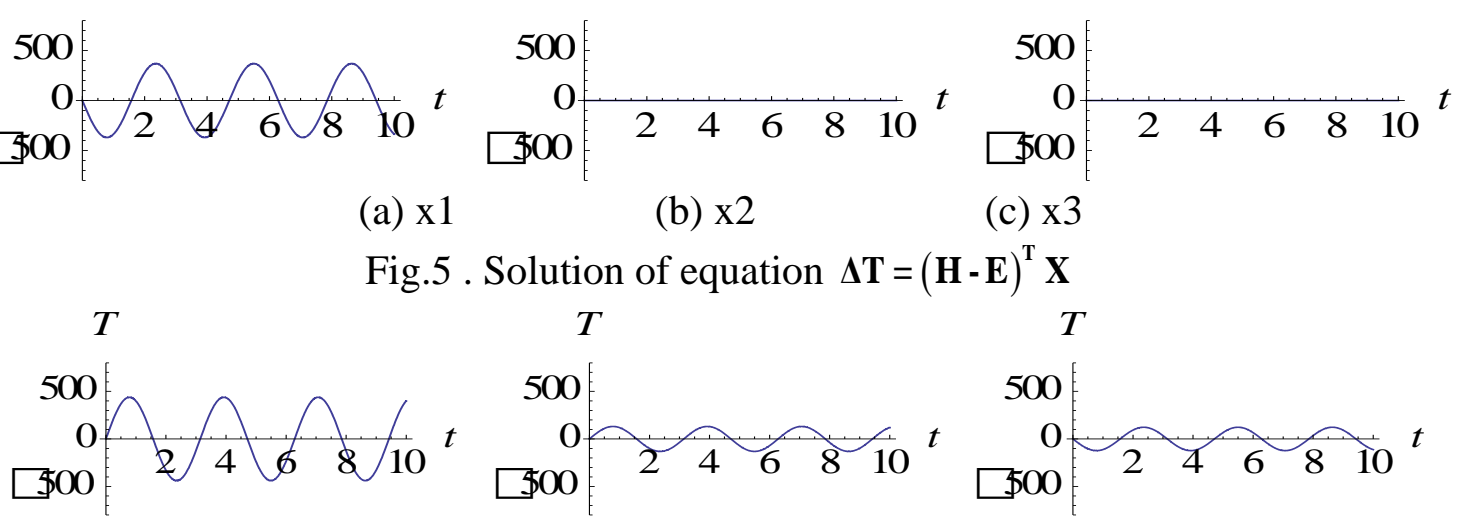

$\begin{array}{ll}\text { (b) } \mathrm{x} 2 & \text { (c) } \mathrm{x} 3 \\ \text { of equation } \Delta \mathrm{T}=(\mathbf{H}-\mathbf{E})^{\mathrm{T}} \mathrm{X}\end{array}$
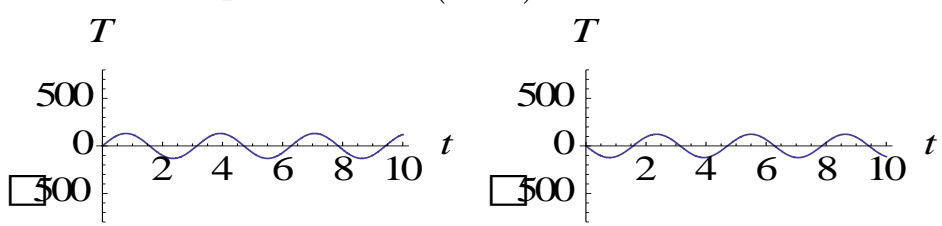
(a) $\mathrm{x} 1$
(b) $\mathrm{x} 2$
(c) $\mathrm{x} 3$
Fig.6 . Solution of equation $\Delta \mathbf{T}=(\mathbf{Q}-\mathbf{H})^{\mathrm{T}} \mathbf{X}$

\section{Conclusion}

This paper has proposed a crack location detection method to the planar 3-RRR parallel compliant mechanism. According to the algorithm shown in Fig. 3, the location of cracked hinge can be identified from the obtained force's variation on PZTs, while theirs elongation remaining the same. The result shows that in order to maintain system's input unchanged, the chain which includes cracked flexure hinge needs much more driving torque than others, which accords with the reality.

\section{Acknowledgments}

The authors are grateful to the China Natural Science Funds (NSFC, Grant No.51175179), and the Fundamental Research Funds for the Central Universities for providing financial support for this work.

\section{References}

[1] Yan, Y.J., et al., Development in vibration-based structural damage detection technique. Mechanical Systems and Signal Processing, 2007. 21(5): p. 2198-2211.

[2] ISERMANN, R. Model based fault detection and diagnosis methods. in Proceedings of the American Control Conference. 1995: Proceedings of the American Control Conference.

[3] Lees, A.W., J.K. Sinha and M.I. Friswell, Model-based identification of rotating machines. Mechanical Systems and Signal Processing, 2009. 23(6): p. 1884-1893.

[4] Midha, A., T.W. Norton and L.L. Howell, On the Nomenclature, Classification, and Abstractions of Compliant Mechanisms. Journal of Mechanical Design, 1994. 116(1): p. 270-279.

[5] Howell, L.L. and A. Midha, A Method for the Design of Compliant Mechanisms With Small-Length Flexural Pivots. Journal of Mechanical Design, 1994. 116(1): p. 280-290.

[6] Zou, J., Kinematic, Dynamics, and Control of Particular Micro-motion System. 2000, University of Saskatchewan: Saskatoon. 
[7] Yong, Y.K., T. Lu and D.C. Handley. Loop closure theory in deriving linear and simple kinematic model for a 3-DOF parallel micromanipulator. in Proceedings of SPIE International Symposium on Microelectronic, MEMS, and Nanotechnology. 2003. Perth, Australia. 